\title{
Revisiting the one in four: the prevalence of psychiatric disorder in the population of England 2000-2014
}

Paul E. Bebbington and Sally McManus

\section{Summary}

Mental health problems are often said to affect one in four people in Britain, although with no consistent explanation of what the figure includes. We used three English national population surveys of psychiatric morbidity from 2000, 2007 and 2014 to provide prevalence rates for recent psychiatric problems. We combined disorders progressively to demonstrate the effects of cumulation. Psychosis had a prevalence of around 1\%, severe common mental disorders added about $8 \%$, and including less-severe common mental disorders gave a value around one in six. The figure of one in four required the inclusion of various other disorders. These values were strikingly stable over the surveys.

\section{Declaration of interest}

None.

\section{Keywords}

Mental disorders; epidemiology; prevalence; temporal change; national survey.

\section{Copyright and usage}

(C) The Royal College of Psychiatrists 2019. This is an Open Access article, distributed under the terms of the Creative Commons Attribution licence (http://creativecommons.org/licenses/by/4.0/) which permits unrestricted re-use, distribution, and reproduction in any medium, provided the original work is properly cited.
The prevalence one in four has been used extensively in service, policy and media contexts to quantify the extent of mental health problems in the British general population. ${ }^{1}$ Ginn and Horder ${ }^{1}$ attempted to identify the origins of this figure and point out a lack of any consistent determination of what the figure should include. It represents a collision between psychiatric epidemiology and the popular understanding of the meaning of mental disturbance: what categories would be acceptable examples of mental disorder to members of the populace? As Ginn and Horder ${ }^{1}$ suggest, the figure has social as well as scientific functions: demonstrating the relevance and significance of the phenomenon of mental illness, encouraging the funding of supportive services, reducing stigma by pointing out its considerable frequency and gaining acceptance of a plausible value. For this reason, any such figure should be examined in relation to its origins and assumptions: there may be conflict between what people know or believe, and what they are being told. There are thus cultural dangers both in overselling and in underselling the frequency of mental illness.

In our view, using an undefined concept risks loss of credibility and the meaning of this figure should therefore be spelt out. The British Adult Psychiatric Morbidity Survey (APMS) programme has applied standardised and structured methods for identifying individual mental disorders in regularly repeated surveys based on randomly chosen samples of the household population. We used the surveys carried out in 2000, 2007 and $2014^{2-4}$ to log both an overall burden of disorder (i.e. the proportion of people with one or more mental health problems) and changes in its magnitude.

\section{Method}

There are difficulties in presenting information about overall prevalence. The first concerns the disorders to be covered. There is little dispute about the inclusion of common mental conditions such as anxiety and depressive disorder, and rarer but severe disorders like psychosis and bipolar disorder. Other disorders recognised in international classifications are also candidates for inclusion, for instance post-traumatic stress disorder (PTSD) and attentiondeficit hyperactivity disorder (ADHD). Likewise, including dependence on illicit drugs and alcohol is unlikely to be contentious. However, in the analyses presented here, we excluded the hazardous use of alcohol and nicotine from analysis because, although clearly a behavioural problem and a social concern, it may not conform to lay ideas of mental disorder. The inclusion of hazardous use would of course result in higher values for the prevalence of mental disorder, to a degree that might be treated with suspicion by potential consumers of this information.

The new analyses presented here are based on the three most recent APM surveys, carried out in 2000, 2007 and 2014. ${ }^{2-4}$ The two later surveys cover only England, and we therefore restrict the analyses of the 2000 survey to respondents from England. Structured interviews were used to provide a logical, valid and clinically meaningful classification, thereby formalising the process of clinical description and case identification. These measures were not adjusted to accommodate intervening changes in the dominant classificatory schemes ${ }^{5,6}$ and are thus comparable across surveys. They are referenced and described in detail in the reports. ${ }^{2-4}$ The values presented here are based on point prevalence for common mental disorders (CMDs) and a prevalence period of up to 1 year for the other disorders. Again, this follows the procedures adopted in the reports.

Comorbidity between psychiatric disorders is common, with the consequence that adding in a new category increases the overall percentage of people affected by less than the individual prevalence of that category. For this reason, we quantified people suffering from mental illness by incorporating disorders sequentially. Such a sequence must inevitably have an arbitrary element. We chose to start with psychotic disorder and CMDs, then moving on to include dependence disorders, as these were quantified identically in all three surveys. Disorders assessed only in the later surveys were included later in the sequence of analysis.

We first identified those suffering from probable psychosis. From the remaining group, we then added people with an identified $\mathrm{CMD}$, in sequence according to their severity. Severity was estimated from their score on the instrument used to identify them, the revised Clinical Interview Schedule. ${ }^{7}$ Scores of 12-17 indicate a significant mental health problem requiring assessment, whereas scores of $\geq 18$ imply treatment is almost certainly needed. From 


\begin{tabular}{|c|c|c|c|c|c|c|c|}
\hline \multirow[b]{3}{*}{ Category } & \multirow[b]{3}{*}{ Disorders } & \multicolumn{6}{|l|}{ Year } \\
\hline & & \multicolumn{2}{|c|}{2000} & \multicolumn{2}{|c|}{2007} & \multicolumn{2}{|l|}{2014} \\
\hline & & $\%$ & $95 \% \mathrm{Cl}$ & $\%$ & $95 \% \mathrm{Cl}$ & $\%$ & $95 \% \mathrm{Cl}$ \\
\hline Severe mental illness & Psychosis in past year & 0.6 & $0.4-0.8$ & 0.5 & $0.3-0.7$ & $1.1^{\mathrm{a}}$ & $0.9-1.4$ \\
\hline Plus severe CMD cases & CIS-R score $\geq 18$ in relation to past week & 8.1 & $7.4-8.8$ & 8.1 & $7.4-8.8$ & 9.0 & $8.2-9.9$ \\
\hline Plus all CMD 'cases' & Current CMDS (CIS-R score $\geq 12$ in relation to past week) & 17.6 & $16.6-18.7$ & 17.0 & $16.0-18.1$ & 18.2 & $17.1-19.3$ \\
\hline Plus substance use disorders & $\begin{array}{l}\text { Harmful/dependent pattern of alcohol or signs of } \\
\text { drug dependence in past year }\end{array}$ & 22.3 & $21.1-23.4$ & 21.0 & $19.9-22.2$ & 22.0 & $20.8-23.2$ \\
\hline Plus developmental disorders & ADHD-positive screen & NA & & 24.5 & $23.3-25.8$ & 26.1 & $24.8-27.4$ \\
\hline Plus personality and other disorders & SAPAS (personality disorder screen), PTSD screen, & NA & & NA & & 31.6 & $30.2-32.9$ \\
\hline
\end{tabular}

bipolar disorder screen

CMD, common mental disorder; CIS-R, Clinical Interview Schedule - Revised; ADHD, attention-deficit hyperactivity disorder; SAPAS, Standardised Assessment of Personality - Abbreviated Scale; PTSD, post-traumatic stress disorder; NA, not available.

Cumulative totals presented. Based on 16- to 74 -year-olds living in England, data weighted to represent the national population. ${ }^{2-4}$ See McManus et al ${ }^{4}$ for details of measures

a. As the screen definition for psychosis includes being on antipsychotic medication, increased prescribing may have been responsible for some of this increase in prevalence.

the remaining sample, we then incorporated people with signs of alcohol or drug dependence. ${ }^{8,9} \mathrm{Next}$, we added those who screened positive for ADHD, ${ }^{10}$ and finally people screening positive for personality disorder, ${ }^{11}$ PTSD ${ }^{12}$ or bipolar disorder. ${ }^{13}$ The ADHD screen was not used until the 2007 survey, and the personality disorder, PTSD and bipolar disorder screens were only introduced in 2014.

\section{Results}

The results are set out in detail in Table 1 . The most severe disorder, psychosis, had a prevalence of around half of $1 \%$ in the two earlier surveys, but this rose significantly to exceed $1 \%$ in 2014. (As the screen definition for psychosis includes being on antipsychotic medication, increased prescribing might have been responsible for this apparent increase in prevalence.) However, the values based on the inclusion of the other types of mental disorder are remarkable for their stability. This is driven to a considerable extent by the virtually unchanging overall frequency of CMDs. Depending on the severity threshold used to include CMDs, combining them with psychosis yields a value of one in ten or one in six. The addition of substance use disorders raises this to just over one in five, and again the values are constant over time. A value of one in four is achieved by adding in the results of screening for ADHD. Finally, incorporating personality and other disorders boosts the figure to around one in three.

\section{Discussion}

We found that psychosis had a prevalence of around $1 \%$, severe CMDs added about $8 \%$ and including less-severe CMDs gave a value around one in six. The figure of one in four required the inclusion of various other disorders.

A major purpose of the APMS programme is to provide information on temporal changes in the prevalence of a range of different disorders, with the expectation that these may be related to a changing social context. Problems in identifying disorders in the general population also arise from the fact that symptoms of mental illness are exponentially distributed. Imposing categories on continuous distributions is always a fraught process. So, for example, although many people have a few isolated affective symptoms, relatively few have sufficient symptoms to justify a diagnosis of mental disorder. ${ }^{14}$ This problem also applies to paranoia ${ }^{15}$ and personality disorder. ${ }^{16}$

There is an inevitable arbitrariness both in selecting the mental conditions for consideration, and in selecting the order in which disorders are entered. The number of disorders determines the number of people who will be identified as suffering from some kind of disorder, whereas the order of entry determines the values of intermediate combinations of disorder. Our choices were based on judgements about how clinicians would probably target treatment in circumstances of comorbidity.

The most striking finding from these analyses is the absence of secular change: for people aged 16-74 years, the prevalence of mental disorders in England over a prolonged period remained remarkably constant. What does this imply? The most parsimonious inference is that the determinants of mental disorder, social or biological, have themselves not varied over that period. However, this would be surprising, certainly given the very considerable changes in social conditions and social behaviour in England since the millennium. A less parsimonious explanation is that there have been changes in a variety of influences bearing on the various disorder types, but that these have tended to cancel each other out. Such hypotheses are open to empirical testing, thereby underlining the importance of programmes like APMS for studying the nature of disorder and the implications for treatment and social policy. For example, changes between the 2014 survey and that planned for 2021 may make it possible to identify the mental health changes occurring in the context of intervening welfare policies characterised by austerity.

Paul E. Bebbington (D, PhD, FRCP, FRCPsych, Emeritus Professor of Social and Community Psychiatry, Division of Psychiatry, Faculty of Brain Sciences, University College London, UK; Sally McManus (D), BA, MSC, Associate, Survey Research Centre, National Centre for Social Research, UK

Correspondence: Prof. Paul Bebbington, Division of Psychiatry, Faculty of Brain Sciences, University College London, 6th Floor Maple House, 149 Tottenham Court Road, London W1T 7NF, UK. Email: p.bebbington@ucl.ac.uk

First received 10 Apr 2019, final revision 19 Jul 2019, accepted 22 Jul 2019

\section{Acknowledgements}

The current analyses were approved by the National Centre for Social Research ethical review committee and are part of independent research funded by the National Institute for Health Research (NHR) Public Health Research Consortium (PHRC) Policy Research Programme 'Establishing trends and understanding context in women's mental health, $1993-2014$ ' PHPEHF50 and not necessarily those of the NIHR or the Department of Health and Social Care. P.E.B. acknowledges the support of University College London Hospital NIHR Biomedical Research Centre.

\section{References}

1 Ginn S, Horder J. 'One in four' with a mental health problem: the anatomy of a statistic. BMJ 2012; 344: e1302.

2 Singleton N, Bumpstead R, O'Brien M, Lee A, Meltzer H. Psychiatric Morbidity Among Adults Living in Private Households. The Stationery Office, 2001.

3 McManus S, Meltzer H, Brugha T, Bebbington P, Jenkins R. Adult Psychiatric Morbidity in England, 2007: Results of a Household Survey. The Health \& Social Care Information Centre, Social Care Statistics, 2009. 
4 McManus S, Bebbington P, Jenkins R, Brugha T (eds). Mental Health and Wellbeing in England: Adult Psychiatric Morbidity Survey 2014. NHS Digital, 2016.

5 American Psychiatric Association. Diagnostic and Statistical Manual of Mental Disorders (5th edn). American Psychiatric Publishing, 2013.

6 World Health Organization (WHO). The ICD-10 Classification of Mental and Behavioural Disorders: Clinical Descriptions and Diagnostic Guidelines. WHO, 1992.

7 Lewis G, Pelosi AJ, Araya R, Dunn G. Measuring psychiatric disorder in the community: a standardised assessment for use by lay interviewers. Psychol Med 1992; 22: 465-86.

8 Saunders JB, Aasland OG, Babor TF, Dela Fuente JR, Grant M. Development of the Alcohol Use Disorders Identification Test (AUDIT): WHO collaborative project on early detection of persons with harmful alcohol consumption, part II. Addiction 1993; 88: 791-804.

9 Drummond C, McBride O, Fear N, Fuller E. Chapter 10: Alcohol dependence. In Mental Health and Wellbeing in England: Adult Psychiatric Morbidity Survey 2014 (eds S McManus, P Bebbington, R Jenkins, T Brugha). NHS Digital, 2016.

10 Kessler RC, Adler L, Ames M, Demler O, Faraone S, Hiripi E, et al. The World Health Organization adult ADHD self-report scale (ASRS): a short screening scale for use in the general population. Psychol Med 2005; 35 : 245-56.

11 Moran P, Leese M, Lee T, Walters P, Thornicroft G, Mann A. Standardised Assessment of Personality - Abbreviated Scale (SAPAS): preliminary validation of a brief screen for personality disorder. Br J Psychiatry 2003; 183 : 228-32.

12 Blanchard EB, Jones-Alexander J, Buckley TC, Forneris CA. Psychometric properties of the PTSD checklist (PCL). Behav Res Ther 1996; 34: 669-73.

13 Hirschfeld RM, Williams JB, Spitzer RL, Calabrese JR, Flynn L, Keck PE, et al. Development and validation of a screening instrument for bipolar spectrum disorder: the Mood Disorder Questionnaire. Am J Psychiatry 2000; 157: $1873-5$

14 Melzer D, Tom BDM, Brugha TS, Fryers T, Meltzer H. Common mental disorder symptom counts in populations: are there distinct case groups above epidemiological cut-offs? Psychol Med 2002; 32: 1195-201.

15 Bebbington PE, McBride O, Steel C, Kuipers E, Radovanovič M, Brugha T, et al. The structure of paranoia in the general population. Br J Psychiatry 2013; 202: 419-27.

16 Yang $\mathrm{M}$, Coid J, Tyrer P. Personality pathology recorded by severity: nationa survey. Br J Psychiatry 2010; 197: 193-9. 